\title{
A study on comparison of hematological, autonomic and respiratory parameters in male drinkers and non- drinkers of Karukutty village, Kerala.
}

\begin{abstract}
Kumar $\mathrm{SS}^{1}$, Angel $\mathrm{J}^{2}$, Elsa $\mathrm{M}^{3}$, Usha $\mathrm{R}^{4}$, Mukkadan $\mathrm{Jk}^{5}$.

The present study was undertaken to compare hematological, autonomic and respiratory parameters in male drinkers and non- drinkers to create awareness and to popularize the knowledge of good/bad effects of drinking. Two hundred and twenty two males with mean age 32 4 were involved in this study comprising of 110 chronic drinkers and 112 non-drinkers, who served as controls. Bleeding time was estimated by Duke Method clotting time was estimated by capillary tube method. Measurement of blood pressure was performed by using sphygmomanometry. Pulse Oximeter was used to measure saturation of hemoglobin $\left(\mathrm{SPO}_{2}\right)$ and pulse rate. The analysis of data was done by SPSS 20.0 . Mean bleeding time was higher in drinkers than non-drinkers. In contrast Clotting time was lower in drinkers. Systolic and diastolic pressure was almost equal in drinkers and non-drinkers. Pulse rate was slightly higher in drinkers than non-drinkers. $\mathrm{SPO}_{2}$ was equal in both drinkers and non-drinkers. We conclude that alcohol prolongs bleeding time and shortens clotting time, however this is contradictory. Alcohol enhances pulse rate may be by decreasing vagal activity and increasing sympathetic activity. Though alcohol causes vasodilation, we haven't observed any change in blood pressure.Inspite of the fact alcohol consumption effects Spo2, no change is observed in spo2 in our study. Hence this study merits continuing with higher sample size.
\end{abstract}

Key words: Drinkers, Non-drinkers.

> CBMJ 2013 July: Vol. 02 No. 02 P: 62-66

\section{Introduction}

Kerala is the highest alcohol consuming state in India. ${ }^{1}$ Researchers has linked alcohol consumption to more than 60 diseases. So Alcohol abuse is becoming a public health concern among the adult males of kerala. Heavy alcohol consumption is associated with detrimental effects on many of the body's systems, as well as with an increased risk of addiction, motor vehicle accidents, trauma, violence, cancer, and suicide. ${ }^{28}$ Excessive alcohol consumption shows toxic effects on the bone marrow; the blood cell precursors; and the mature red blood cells (RBC's), white blood cells (WBC's), platelets and plasma proteins. Heavy drinking can cause the number of oxygen-carrying red blood cells to be abnormally low. This condition, known as anemia, can trigger a host of symptoms, including fatigue, shortness of breath, and lightheadedness. Heavy drinking makes platelets more likely to clump together into blood clots, which can lead to heart attack or stroke. ${ }^{2}$ A decrease in the number and function of WBC's increases the drinker's risk of serious infection, and impaired platelet production and function interfere with blood clotting, leading to symptoms ranging from a simple nosebleed to bleeding in the brain. ${ }^{3,4}$ Large amounts of alcohol are known to increase blood pressure however different drinking habits seem to have different effects on blood pressure. ${ }^{5}$ It was reported that there is a level of alcohol consumption, of approximately four drinks per day, below which drinkers have either similar or lower blood pressure levels compared to nondrinkers. ${ }^{6}$
However, there were no enough studies from India, revealing the association of alcohol consumption with BP and hypertension. ${ }^{24}$

Alcohol consumption, even at very modest intake levels, is associated with less lung restriction. Moderate alcohol drinkers may have better lung airways function than nondrinkers but heavier drinkers had worse function. ${ }^{8}$ It was reported that there was also no evidence of an association between consumption of alcohol and airway restriction. ${ }^{9}$

1. ** Sai sailesh kumar.G

Research Scholar, LFMRC,

Angamaly. Kerala, India.

2. Jose Angel

PG Students

Department of Physiology

LIMSAR, Angamaly, Kerala, India

3. Mathew Elsa

PG Students

Department of Physiology

LIMSAR, Angamaly, Kerala, India

4. Rose Usha

Associate Professor

Department of Anatomy, LIMSAR, Angamaly.

Kerala, India

5. *Dr.J.K.Mukkadan

Research Director,

Little Flower Medical Research Centre (LFMRC), Angamaly. Kerala, India.

\footnotetext{
* Address of correspondence

E-mail: ** Saisailesh.kumar@gmail.com *drmukkadan@sify.com
} 
... Original Article

Investigations of large-scale observational cohorts have suggested that light to moderate drinking may be associated with decreased mortality rates and with decreased risk of cardiovascular disease. ${ }^{14}$ The present study was undertaken to compare hematological, autonomic and respiratory parameters in male drinkers and non- drinkers to create awareness and to popularize the knowledge of $\mathrm{good} / \mathrm{bad}$ effects of drinking.

\section{Methods}

\section{Study design and participants}

The present study was conducted in the department of physiology in Little Flower Medical Research Centre, Angamaly, Kerala, India. Two hundred and twenty two males with mean age $32 \pm 4$ were involved in this study comprising of 110 chronic drinkers and 112 non-drinkers, who served as controls. Subjects who were consuming alcohol for at least 5 years were designated as drinkers and strictly non-alcoholic drinks consumers were designated as control. The purpose and procedure of the study were explained to each subject. Written informed consent was taken from all the participants. Study protocol was approved by Institutional Ethics Committee of Little Flower Medical Research Centre, Angamaly.

\section{Hematological parameters}

Bleeding time was estimated by Duke Method clotting time was estimated by capillary tube method. ${ }^{10}$ In duke method, a puncture is made on finger tip and the time recorded when blood first appears. The blood is carefully blotted every 30 seconds, with care begin taken that the wound site is not touched. The time is recorded when bleeding stops. The time interval from onset of bleeding to stoppage of bleeding should be reported as bleeding time. The Capillary Tube Method involves collecting blood in a capillary tube that does not contain anticoagulant. The timer is started when the blood first enters the tube. The outside of the tube is carefully wiped and every 30 seconds a piece of the tube is broken. The time is recorded when a strand of fibrin appears between the two pieces of capillary tube. ${ }^{11}$

\section{Autonomic and respiratory parameters}

Measurement of blood pressure was performed by using sphygmomanometry. ${ }^{12}$ Pulse Oximeter is used to measure saturation of hemoglobin and pulse rate. Pulse Oximetry is a non-invasive method allowing the monitoring of the saturation of hemoglobin and pulse rate. The Oximeter uses oximetry to measure functional oxygen saturation in blood. Pulse Oximeter works by applying the sensor to a pulsating arteriolar vascular bed, such as a finger or toe. The sensor contains dual light source and a photonic detector. Bone, tissue, pigmentation, and venous vessels normally absorb a constant amount of light over time. The arteriolar bed normally pulsates and absorbs variable amounts of light during the pulsations. The ratio of light absorbed is translated into a measurement of functional oxygen saturation (SpO2). Because a measurement of $\mathrm{SpO} 2$ is depend upon light from the sensor, excessive ambient light can interfere with this measurement.

Pulse Oximetry is based on two principles

- Oxyhaemoglobin and deoxyhaemoglobin differ in their absorption of red and infrared light.

- The volume of arterial blood in tissue (hence light absorption by the blood) changes during the pulse.

The Oximeter determines $\mathrm{SpO} 2$ by passing red and infrared light into an arteriolar bed and measuring changes in light absorption during pulsatile cycle. Red and infrared low - voltage light emitting diodes (LED) serves as light sources; a photonic diode serves as the photodetector. Because oxyhaemoglobin and deoxyhaemoglobin differ in light absorption, the amount of red and infrared light absorbed by blood is related to hemoglobin oxygen saturation. To identify the oxygen saturation of arterial hemoglobin, the Oximeter uses the pulsatile nature of arterial flow. During systole, a new pulse of arterial blood enters the vascular bed, and blood volume and light absorption increase. During diastole, blood volume and light absorption reach their lowest point. The Oximeter bases its SpO2 measurements on the difference between maximum and minimum absorption (measurements at systole and diastole).by doing so, it focuses on light absorption by pulsatile arterial blood, eliminating the effects of non - pulsatile absorption by tissue, bone and venous blood.

\section{Data collection}

The preset study was conducted at 9 am in the morning for the convenience of subjects. Two investigators of our study performed the experiment and collected data from the student at the same time.

Data analysis

The analysis of data is done by SPSS 20.0. Independent sample t test is used for data analysis. significant level ( $p$ value) is set as $5 \%$.

\section{Results}

The analysis of data is presented in Table no: 1. Mean bleeding time is higher in drinkers $(81.8 \pm 38.10$ $\mathrm{sec})$ than non-drinkers $(71.4 \pm 35.28 \mathrm{sec})$ (Figure no: $1)$. However this difference is statistically not significant ( $p$ value 0.16 ). In contrast Clotting time is lower in drinkers $(262.46 \pm 84.89 \mathrm{sec})$ than nondrinkers (264.26 $\pm 94.72 \mathrm{sec}$ ) (Figure no: 2$)$, which is not statistically significant ( $p$ value 0.92 ). Systolic and diastolic pressure is almost equal in drinkers and non- 
drinkers (Figure no: 3, Figure no: 4). Pulse rate is slightly higher in drinkers $(92.58 \pm 2.60 \mathrm{~min})$ than nondrinkers (89.3 \pm 11.35$)$ (Figure no: 5). However this variation is statistically not significant ( $p$ value 0.049 ).

$\mathrm{SPO}_{2}$ (saturation of hemoglobin) is equal in both drinkers and non-drinkers (Figure no: 6).

\begin{tabular}{|l|l|l|l|}
\hline & Drinkers & Non-Drinkers & $p$ value \\
\hline $\begin{array}{l}\text { BT (in } \\
\text { seconds) }\end{array}$ & $81.8 \pm 38.10$ & $71.4 \pm 35.28$ & 0.16 \\
\hline $\begin{array}{l}\text { CT (in } \\
\text { seconds) }\end{array}$ & $262.46 \pm 84.89$ & $264.26 \pm 94.72$ & 0.92 \\
\hline $\begin{array}{l}\text { SBP } \\
\text { (mmHg) }\end{array}$ & $115.8 \pm 7.85$ & $115.64 \pm 13.02$ & 0.941 \\
\hline $\begin{array}{l}\text { DBP } \\
\text { (mmHg) }\end{array}$ & $80.2 \pm 7.42$ & $80.02 \pm 8.57$ & 0.911 \\
\hline $\begin{array}{l}\text { PR } \\
\text { (minutes) }\end{array}$ & $92.58 \pm 2.60$ & $89.3 \pm 11.35$ & 0.049 \\
\hline SPo2 (\%) & $0.99 \pm 0.01$ & $0.99 \pm 0.01$ & 0.574 \\
\hline
\end{tabular}

Table no: 1 Mean values of hematological, autonomic and respiratory parameters in male drinkers and nondrinkers

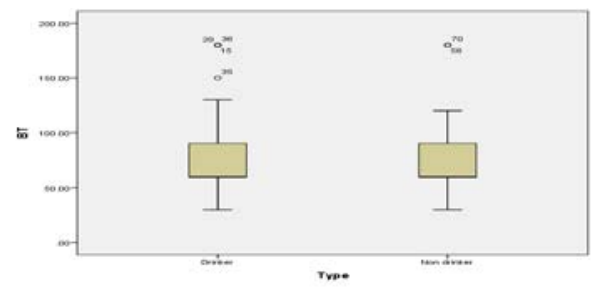

Figure no: 1 Mean bleeding time in drinkers and non drinkers.

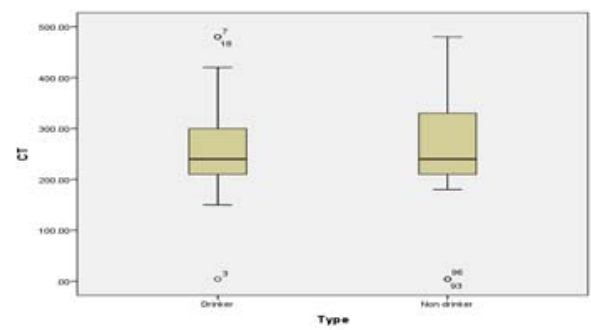

Figure no: 2 Mean clotting time in drinkers and non drinkers.

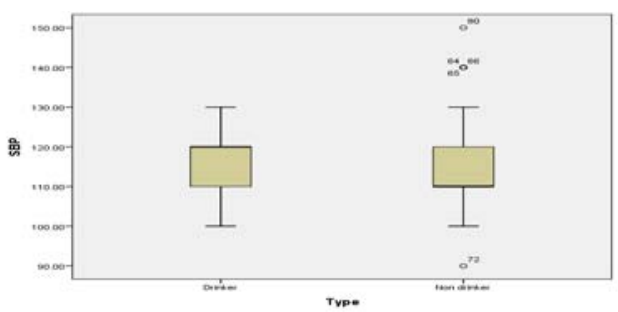

Figure no: 3 Mean systolic blood pressure in drinkers and non drinkers.

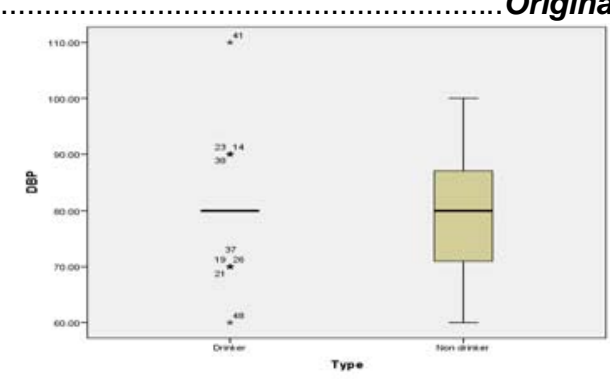

Figure no: 4 Mean diastolic blood pressure in drinkers and non drinkers.

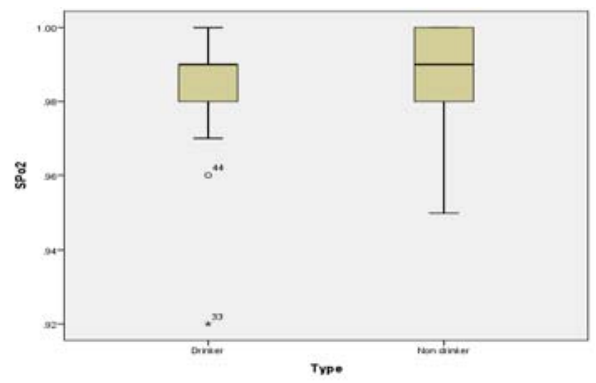

Figure no: 5 Mean pulse rate in drinkers and non drinkers.

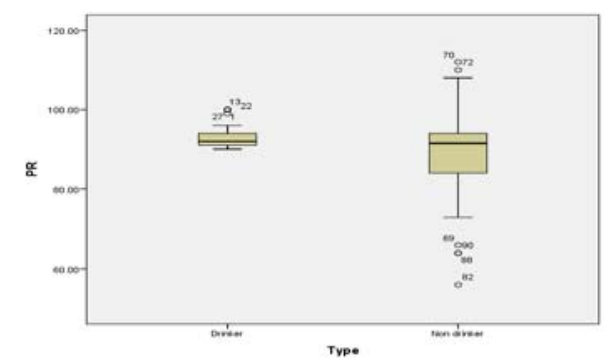

Figure no: 6 Mean $\mathrm{Spo}_{2}$ in drinkers and non drinkers

\section{Discussion}

Alcohol decreases platelet numbers in the blood (thrombocytopenia) and also impaired platelet function (thrombocytopathy). ${ }^{13}$ We agree with this study as we have observed higher bleeding time in drinkers than non-drinkers. However, alcohol-related thrombocytopenia generally is transient, and platelet counts usually return to normal within 1 week of abstinence. The exact mechanisms underlying alcohol-related thrombocytopenia remain unknown. Some researchers have suggested that alcohol intoxication itself, rather than alcohol-related nutritional deficiencies, causes the decrease in platelet numbers. ${ }^{4}$ Alcohol intake has been shown to have serious effects on some haematological parameters which could predispose heavy alcohol consumers to infections and bone marrow malfunctions. ${ }^{14}$ The results of the various studies of 
the effects of ethanol on human platelets are contradictory. ${ }^{15}$

Light-to-moderate alcohol consumption is associated with lower levels of coagulatory factors, but higher intake is associated with impaired fibrinolytic potential. ${ }^{16}$ The factor VII concentration was lowest in subjects who consumed the highest amount of alcohol. ${ }^{15}$ An increase in alcohol consumption from less than 1 drink per day to more than 5 drinks per day caused a significant increase in factor IX levels with no effect on factor VII and factor VIII levels. On the other hand, the increase in alcohol intake leads to decrease in plasma fibrinogen concentration. ${ }^{17}$ Fibrinogen rose less in persons who became drinkers or remained drinkers and, interestingly, increased more in persons who quit drinking. ${ }^{18}$ In the present study we have observed slightly lower clotting time in drinkers.

A moderate intake of wine is associated with a lower risk of mortality from all causes in persons with hypertension. ${ }^{19}$ However heavy alcohol consumption has an important effect on BP, and thus cessation of alcohol consumption must be recommended as a priority for hypertensive alcohol drinkers. ${ }^{20}$ Alcohol consumption has been shown to be a risk factor for high $\mathrm{Bp},{ }^{21,22}$ an effect which is reversible. ${ }^{23}$ It was reported that there is a level of alcohol consumption, of approximately four drinks per day, below which drinkers have either similar or lower blood pressure levels compared to nondrinkers. ${ }^{6}$ Some groups recorded higher levels of systolic and diastolic BP among alcohol drinkers than non-drinkers, and in others it was reverse. However there is no consistent association of alcohol consumption with blood pressure. ${ }^{24}$.In the present study we haven't observed any notable difference in systolic and diastolic blood pressure between drinkers and non-drinkers. Blood pressure is not increased in drinkers probably because of vasodilator effects of alcohol. ${ }^{29}$

It was reported that alcohol consumption increases heart rate. ${ }^{25}$ In contrast some studies suggested that alcohol consumption is associated with reduced vagal activity. ${ }^{26}$ The reason for a positive association between alcohol intake and heart rate is unclear but possibilities include an increase in sympathetic activity secondary to vasodilation or increased calcium entering into cardiac myocytes. ${ }^{27}$ Acute increases in plasma alcohol increase heart rate and sympathetic nerve activity. ${ }^{29}$ We agree with these reports as we have observed higher pulse rate in drinkers.

Alcohol may reduce the affinity of hemoglobin for oxygen. The reason for this effect is unknown. It is possible that the fall in blood $\mathrm{pH}$, though very small, may be implicated. ${ }^{29}$ Drinking alcohol before retiring resulted in lower arterial blood oxygen saturation
$\left(\mathrm{SpO}_{2}\right)$ during the early half of sleep when compared to the values on the alcohol-free day and drinking alcohol with dinner. ${ }^{30}$ However we haven't observed any notable difference in $\mathrm{SpO}_{2}$ between drinkers and non-drinkers.

\section{Conclusion}

We conclude that alcohol prolongs bleeding time and shortens clotting time, however this is contradictory. Alcohol enhances pulse rate may be by decreasing vagal activity and increasing sympathetic activity. Though alcohol causes vasodilation, we haven't observed any change in blood pressure.Inspite of the fact alcohol consumption effects Spo2, no change is observed in spo2 in our study. Hence this study merits to continue with higher sample size.

\section{References:}

1. http://www.indianexpress.com/news/kerala-at-top-jk-bottom-inindia-s-first-alcohol-atlas/302830.

2. http://www.webmd.com/mental-health/alcoholabuse/features/12-health-risks-of-chronic-heavy-drinking

3. $G W M c$ Garry, S Gatehouse, J Hinnie. Relation between alcohol and nose bleeds. BMJ. 1994; 309:640.

4. Harold s. Ballard, M.D. The hematological complications of alcoholism. Alcohol Health \& Research world. 1997; 21(1): 4252.

5. Seppä K, Laippala P, Sillanaukee P. Drinking pattern and blood pressure. Am J Hypertens. 1994 Mar; 7(3): 249-54.

6. Jackson R, Stewart A, Beaglehole R, Scragg R. Alcohol consumption and blood pressure. Am J Epidemiol. 1985 Dec; 122(6): 1037-44

7. Sisson JH, Stoner JA, Romberger DJ, Spurzem JR, Wyatt TA, Owens-Ream J, Mannino DM. Alcohol intake is associated with altered pulmonary function. Alcohol. 2005 May; 36(1):1 930.

8. Stanton $T$ Siu, MD, Natalia Udaltsova, PhD and Arthur $L$ Klatsky, MD. Alcohol and lung airways function. Perm J. 2010 spring; 14(1): 11-18.

9. Cohen BH, Celentano DD, Chase GA, Diamond EL, Graves CG, Levy DA, Menkes HA, Meyer MB, Permutt S, Tockman MS. Alcohol consumption and airway obstruction. Am Rev Respir Dis. 1980 Feb; 121(2):205-15.

10. Ghai, C.L. A Textbook of Practical Physiology. 5th Edn, Jaypee Brothers, New Delhi, Haematology 1999; 84-101.

11. http://loudoun.nvcc.edu/vetonline/vet131/hemostasis.htm.

12. Dorothee Perloff, Carlene Grim, John Flack, Edward D. Frohlich, Martha Hill, Mary McDonald and Bruce Z. Morgenstern. Human blood pressure determination. Circulation. 1993; 88: 2460-2470.

13. Cowan DH. Effect of alcohol on haemostasis. Semin Hematol.1980;17: 137-47.

14. E.O. Akanni, V.O. Mabayoje, T.O. Zakariyahu and D.P. Oparinde. Haematological Characterization among Heavy Alcohol Consumers in Osogbo Metropolis. Research Journal of Medical Sciences. 2010; 4(2): 48-52.

15. Raneem O. Salem, $P h D$, and Michael Laposata, MD, PhD. Effects of Alcohol on Hemostasis. Am J Clin Pathol 2005; 123 (Suppl 1): S96-S10.

16. Kenneth J. Mukamal, Praveen p. Jadhav, Ralph B. D'Agostino, Joseph M. Massaro, Murray A. Mittleman, Izabella Lipinska et al., Alcohol consumption and hemostatic factors; Analysis of the Framingham offspring cohort. Circulation. 2001; 104: 13671373. 
17. Wannamethee SG, Lowe GD, Shaper G, et al. The effects of different alcoholic drinks on lipids, insulin and haemostatic and inflammatory markers in older men. Thromb Haemost.2003;90: 1080-1087.

18. Tochi M Okwuosa, Oana Klein, Cheeling Chan, Pamela Schreiner, Kiang Liu, David Green. Longterm change in alcohol consumption status and variations in fibrinogen levels; the coronary artery risk development in young adults(CARDIA) study. BMJ open. 2013; e002944.

19. Serge C Renaud, René Guéguen, Pascale Conard, Dominique Lanzmann-Petithory Jean-Marc Orgogozo, and Olivier Henry. Moderate wine drinkers have lower hypertension-related mortality: a prospective cohort study in French men $^{1-3}$. Am J Clin Nutr. 2004; 80: 621-5.

20. María Teresa Aguilera, Alejandro de la Sierra, Antonio Coca, Ramo'n Estruch, Joaquín Ferna'ndez-Sola', Alvaro UrbanoMa'rquez. Effect of Alcohol Abstinence on Blood Pressure Assessment by 24-Hour Ambulatory Blood Pressure Monitoring. Hypertension. 1999; 33: 653-657.

21. Klatsky AL, Friedman GD, Armstrong MA. The relationships between alcoholic beverage use and other traits to blood pressure: a new Kaiser Permanente study. Circulation. 1986; 73: 628-36.

22. Klatsky AL. Alcohol and blood pressure. In: Laragh JH, Brenner $B M$, eds. Hypertension: Pathophysiology, diagnosis and treatnment. New York: Raven Press, 1990; 277-94.
23. Potter JF, Beevers DG. Pressor effect of alcohol in hypertension. Lancet 1984; 1: 119-22.

24. Yadlapalli Sriparvati Kusuma, Bontha Veerraju Babu, Jammigumpula Masthannaiah Naidu. Association of alcohol consumption and blood pressure in some low socioeconomic groups from Andhra pradesh, India. Coll. Antropol. 2009; 33(2): 417-422.

25. J $M$ Ryan and $L$ G Howes. Relations between alcohol consumption, heart rate and heart rate variability in men. Heart. 2002 December; 88(6): 641-642.

26. Pellizzer AM, Kamen PW, Esler MD, et al. Comparative effects of mibefradil and nifedipine gastrointestinal transport system on autonomic function in patients with mild to moderate essential hypertension. J Hypertens. 2001; 19: 279-85.

27. Howes LG, Reid JL. The effects of alcohol on local, neural and humoral cardiovascular regulations. Clin Sci. 1985; 71:9-15.

28. Jason Andrade, Ken G. Gin. Alcohol and the heart. BCMJ, June 2009; 51(5): 200-205

29. Philippe van de Borne, Allyn M. Mark, Nicola Montano, Decio Mion, Virend K. Somers. Effect of alcohol on sympathetic activity, hemodynamics and chemoreflex sensitivity. Hyper tension. 1997; 29: 1278 - 1283

30. Ichiro Izumi, Ali Nasermoaddeli Michikazu Sekine, and Sadanobu Kagamimori. Effect of moderate alcohol intake on nocturnal sleep respiratory parameters in healthy middle- aged men. Environ Health Prev Med. 2005 January; 10 (1): 16-20. 